\title{
Indoor Navigation using LIFI
}

\author{
Prof. B. B. Gite ${ }^{1}$, Pankaj Maydeo ${ }^{2}$, Shubhangi Bade ${ }^{3}$, Tushar Muluk ${ }^{4}$ \\ Head of Dept., Computer Dept., SAOE, Kondhwa, Pune, India ${ }^{1}$ \\ Student, Computer Dept., SAOE, Kondhwa, Pune, India ${ }^{2,3,4}$
}

\begin{abstract}
The LI-FI is the most up to date innovation in the Field of remote correspondence. These days many individuals are utilizing web to satisfy their undertaking through wired or remote. As the quantity of clients is expands the rate of information transmission in the remote system gets consequently diminishes. WI-FI gives us speed close around $150 \mathrm{mbps} .11 \mathrm{n}$ yet steel it is not ready to satisfy the necessity of the client due to such reason we are presenting the LI-FI. As per the German physicist Harald Hass LI-FI gives more speed (10megabits every second) information transmission by utilizing obvious light. So here in this condition we are dissect the LI-FI/WI-FI.It's a similar thought band behind infrared remote controls yet significantly more capable. Haas says his innovation, which he calls DLIGHT, can create information rates speedier than our normal broadband association. In this we will look at and broke down the speed of LI-FI and WI-FI and furthermore organize sticking issue amid the expansion the expanding of clients.
\end{abstract}

Keywords: Wi-Fi, Radio Spectrum, Li-Fi, Visible Spectrum.

\section{INTRODUCTION}

Li-Fi can be considered as a light-primarily based Wi-Fi. The more we become dependent upon 'the cloud' or our That is, it makes use of light rather than radio waves to own 'media servers' to store all of our files, including transmit records and instead of Wi-Fi modems, Li-Fi could movies, music, pictures and games, the more we will want utilize handset -fitted led lighting that can light a room bandwidth and speed. Therefore RF-based technologies and in addition transmit and get data.

Li-Fi is probably another model for optical faraway innovation or the higher learning than make unexampled belonging inner a limited little sector for the specific situation. Growing requests are meant for unmatched switch speeds, is to get to snappier and more at ease statistics transmission furthermore as ecological and without a doubt human neighbourly innovation. Li-Fi will soak up for lighting administrations as well as also a universe of recent and super spearheading administrations and out coming best results. The lighting fixtures administration makes improve precise outcomes for the far off facts correspondence the fine association is that, we are able to make the earth mercury loose and use it within the sheltered way. With this mercury environment we are able to even produce some other innovation anywhere all through the sector.

\section{LITERATURE SURVEY}

Most of us are familiar with Wi-Fi (Wireless Fidelity), which uses $2.4-5 \mathrm{GHz}$ RF to deliver wireless Internet access around our homes, schools, offices and in public places. We have become quite dependent upon this nearly ubiquitous service. But like most technologies, it has its limitations. While Wi-Fi can cover an entire house, its bandwidth is typically limited to 50-100 megabits per second (Mbps) today using the IEEE802.11n standard. This is a good match to the speed of most current Internet services, but insufficient for moving large data files like HDTV movies, music libraries and video games. such as today's Wi-Fi are not the optimal way. In addition, Wi-Fi may not be the most efficient way to provide new desired capabilities such as precision indoor positioning and gesture recognition. Optical wireless technologies, sometimes called visible light communication (VLC), and more recently referred to as Li-Fi (Light Fidelity), on the other hand, offer an entirely new paradigm in wireless technologies in terms of communication speed, flexibility and usability.

\section{ARCHITECTURAL DESIGN}

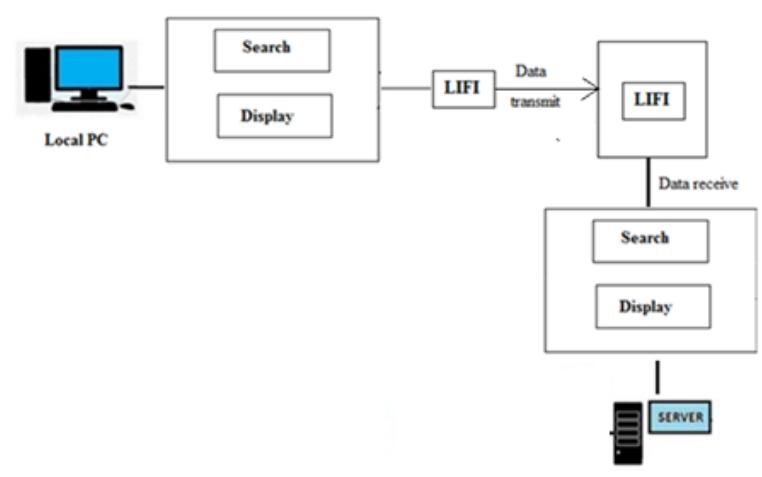

\section{PROPOSED SYSTEM}

Li-Fi (Light Fidelity) is a high speed, wireless communication using visible light. It falls under the category of optical wireless communications. Data transmission takes place through LED bulbs whose 
intensity varies. Based on this variation, communication occurs digitally. The word Li-Fi was first coined by Harald Haas at the University of Edinburgh. This technology has vast applications where the use of $\mathrm{Wi}-\mathrm{Fi}$ is limited or banned. It also takes out the adverse health effects of using electromagnetic waves. Unless light is seen, data can't be hacked and so data transmission is secure. Data transmission is typically in terms of Giga bytes per second.

As light is present everywhere it would be very convenient if we channel this into transmitting data. This is where Li-Fi (Light Fidelity) technology comes into play. In Li-Fi, Digital data transmission takes place using LED bulbs with flickering intensity controlled by varying currents. The working is simple as shown in Fig. 1. There is a light emitter at one end. Light emitter can be simply LED. When the LED is on, a digital ' 1 ' is transmitted. When the LED is off, a digital ' 0 ' is transmitted. This is received by a photo detector at the other end.

This refers to a single bit data transmission. An array of LED's can be connected so that a large amount of data transmission takes place. Speed depends on the rate of variation of LED intensity. In this manner data is encoded and transmitted. The main factors to be considered during design are -

Line of Sight (LoS).

Presence of Light.

Illumination.

Li-Fi provides with more bandwidth that is free and clean to use. It is also unlicensed. Li-Fi additionally presents with extra information density compared to that of $\mathrm{Wi}-\mathrm{Fi}$. The statistics density is about a thousand times than wireless. This is due to less interference of mild than RF waves. Because of high facts density and bandwidth the output pace is likewise very high.

\section{ADVANTAGE}

Li-Fi can be used around highly inflammable areas like Petrol bunks, aircrafts etc. as there wouldn't be any interference with inflammable objects. It can be used for underwater communication as light passes through almost all liquid media. Li-Fi provides an environment free from electromagnetic waves and thus creates a healthy environment. Li-Fi technology provides safety, security, fast and efficient communication. The Electromagnetic free environment created by Li-Fi can be used for hospital applications.

\section{CONCLUSION}

With the developing technology and growing use of the internet offerings, opportunities are very excessive that use of li-fi era might be quickly in practice. The idea of li-fi is spreading so fast as it is simple to use, it's miles attracting interest of human beings. The usage of li-fi era offers a totally golden opportunity to replace or to present alternative to the radio based wi-fi technologies. As the quantity of people and the access of internet is increasing on one of these large scale, getting access to net through wireless will soon be insufficient as the utilization is growing however the bandwidth stays the identical. On this record paper we finish that the opportunities are numerous and may be explored in addition this technology is in manufacturing system to provide each bulb to become a wi-fi hotspot to transmit wireless statistics.

\section{ACKNOWLEDGMENT}

We would like to Prof. B.B. Gite thank Sinhgad Academy of Engineering Kondhawa, Department of Computer for supporting and guiding us.

\section{REFERENCES}

[1] J Vittahal S Saptasagare, "Next of Wi-Fi an Future Technology in Wireless Networking Li-Fi Using Led Over Internet of Things," International Journal of Emerging Research in Management and Technology, Volume 3, Issue 3, March 2014, ISSN: 2278-9359.

[2] Ravi Prakash and Prachi Agarwal, "The New Era of Transmission and Communication Technology: Li-Fi (Light Fidelity) LED \& TED Based Approach," International Journal of Advanced Research in Computing Engineering and Technology, Volume 3, Issue 2, Febraury 2014, ISSN: 2278-1323.

[3] DhakaneVikasNivrutti and Ravi RamchandraNimbalkar, "Light Fidelity: A Reconnaissance of Future Technology," International Journal of Advanced Research in Computer Science and Software Engineering, Volume 3, Issue 11, November 2013, ISSN: 2277128X.

[4] Jay H.Bhut, Dharmrajsinh N. Parmar, Khushbu V. Mehta, "LI-FI Technology - A Visible Light Communication," International Journal of Engineering Development and Research, ISSN: 23219939.

[5] Rahul R. Sharma, Raunak, AkshaySanganal, "Li-Fi Technology," International Journal of Computer Technology and Applications, Vol 5(1), 150-154, ISSN: 2229-6093.

[6] http://www.slideshare.net/shwrvppt/li-fi-tch.

[7] https://en.wikipedia.org/wiki/Li-Fi.

[8] http://purelifi.com/news_media/lifi-white-papers/ 